Article

\title{
Effective Conformal Descriptions of Black Hole Entropy
}

\section{Steven Carlip}

Department of Physics, University of California at Davis, Davis, CA 95616, USA;

E-Mail: carlip@physics.ucdavis.edu.

Received: 1 July 2011; in revised form: 12 July 2011 / Accepted: 19 July 2011 /

Published: 20 July 2011

\begin{abstract}
It is no longer considered surprising that black holes have temperatures and entropies. What remains surprising, though, is the universality of these thermodynamic properties: their exceptionally simple and general form, and the fact that they can be derived from many very different descriptions of the underlying microscopic degrees of freedom. I review the proposal that this universality arises from an approximate conformal symmetry, which permits an effective "conformal dual" description that is largely independent of the microscopic details.
\end{abstract}

Keywords: black holes; Bekenstein-Hawking entropy; conformal dual

\section{Introduction}

Since the seminal work of Bekenstein [1] and Hawking [2], we have understood that black holes behave as thermodynamic systems, with characteristic temperatures and entropies. A central task for quantum gravity is finding a statistical mechanical description of this behavior in terms of microscopic states. One important clue may come from the surprising "universality" of these thermodynamic properties, which manifests itself in two ways:

- The Bekenstein-Hawking entropy

$$
S=\frac{\mathcal{A}}{4 \hbar G}
$$

takes the same simple form for all black holes, independent of the charges, spin, and even the number of spacetime dimensions. If the action differs from that of general relativity, the entropy is modified, but again in a simple and universal way [3]. 
- While the "correct" microscopic origin of black hole entropy is not settled, we now have a number of candidates, coming from string theory, loop quantum gravity, holographic entanglement entropy, Sakharov-style induced gravity, and several other approaches. (See [4] for a more detailed discussion.) But although these methods count very different states-or, in the case of Hawking's original derivation [2], seem to know nothing about quantum gravitational states at all-they all yield the same entropy. To be sure, no approach is yet complete, and each requires additional assumptions. But within its domain of validity, each seems to work fairly well, and the universal agreement with (1) remains deeply mysterious.

One attempt to explain this universality is to posit that black hole thermodynamics has an effective "dual" description in terms of a two-dimensional conformal theory, whose key properties are governed by symmetries. Black holes are, of course, neither two-dimensional nor conformally invariant, so at first sight this proposal seems rather surprising. But in the sixteen years or so since such a description was first proposed [5], the idea has become considerably more plausible. In special cases-in particular, the (2+1)-dimensional BTZ black hole [6] - the two-dimensional conformal description seems quite convincing [7-9], although subtle questions about the conformal field theory remain [10,11]. For more general black holes [12-14], the issue is much less settled, but the recent discovery of an extremal Kerr/CFT correspondence [15] has brought a good deal of renewed interest.

In this paper, I will review the arguments for a two-dimensional conformal description-a CFT dual, in the current string-theory-inspired language-for a variety of black holes. I will start by sketching the general derivation of such a dual, then describe a few examples, and finally suggest a physical explanation for the Bekenstein-Hawking entropy. My purpose is not to explain any specific instance of this construction in depth; for that, the reader should refer to the primary literature. Rather, I will try to describe the general framework in which to think about CFT duals of black holes.

\section{Building a Black Hole/CFT Correspondence in Four Easy Steps}

While the details differ, most derivations of black hole/CFT duality are based on the same four steps:

i. Find an appropriate boundary and impose boundary conditions that specify properties of the black hole;

ii. Determine how these boundary conditions affect the symmetries of general relativity, the algebra of diffeomorphisms or "surface deformations";

iii. Look for a preferred subalgebra of diffeomorphisms of the circle (Diff $S^{1}$ ), or perhaps two copies (Diff $\left.S^{1} \times \operatorname{Diff} S^{1}\right)$;

iv. Use standard methods from conformal field theory—in particular, the Cardy formula [16,17]— to extract physical information from this symmetry.

I will explain each of these in turn. 


\subsection{Find an Appropriate Boundary and Impose Boundary Conditions}

A question about a quantum black hole is almost always a question about conditional probabilities: "If a black hole with certain characteristics is present, what is the probability that ...?" One must therefore decide how to impose the conditions, that is, the presence and specific characteristics of the black hole. Although there has been some work on adding these conditions as explicit constraints [18,19], probably the easiest approach is probably to impose them as boundary conditions.

This is most easily done for the (2+1)-dimensional black hole of Bañados, Teitelboim, and Zanelli (BTZ) [6]. This black hole lives in an asymptotically anti-de Sitter spacetime, and as Brown and Henneaux showed [20], the asymptotic boundary conditions at infinity determine its mass and spin. Many near-extremal black holes in more than three spacetime dimensions have a near-horizon region that closely resembles (2+1)-dimensional anti-de Sitter space [7,21], and Brown-Henneaux boundary conditions can be imposed at the "boundary" of this region. Similarly, extremal Kerr black holes have a near-horizon region that looks like anti-de Sitter space [22], and one can impose modified AdS-like boundary conditions there [15].

An alternative, and perhaps more intuitive, choice is to treat the event horizon itself as a boundary [12-14]. The horizon is not, of course, a real boundary: an infalling observer can pass through it freely. It is, however, a place at which one can restrict the metric to impose the condition that a particular type of black hole be present.

Such a restriction is essentially a boundary condition. In the path integral formalism, for instance, it amounts to splitting spacetime into two pieces along the horizon, with separate path integrals for each piece, each with a suitable boundary condition. For two-dimensional free field theories [23] and three-dimensional Chern-Simons theory [24], such splittings have been investigated carefully, and it has been shown that one can reconstruct the full path integral by "gluing" the two halves and integrating over boundary data. This last integration can be viewed as a quantum mechanical sum over a complete set of intermediate states; the role of the boundary conditions is to determine which set of intermediate states appears.

For quantum gravity, the imposition of boundary conditions at the true horizon is difficult, essentially because the horizon is a null surface. In practice, one must instead impose boundary conditions on a "stretched horizon" [25], a timelike surface just outside the horizon, and then take a limit as the surface shrinks to the horizon. This is not an entirely satisfactory approach, though - the results sometimes depend on the limiting procedure [26] — and there is clearly room for improvement.

\subsection{Determine how These Boundary Conditions Affect the Symmetries}

In its Hamiltonian form, general relativity in $n$ spacetime dimensions is a constrained theory, with $n$ first-class constraints $\mathcal{H}_{\mu}$ [27]. These constraints generate the symmetries of the theory. In the covariant phase space formalism [28-30], these symmetries are the spacetime diffeomorphisms. That is, for a diffeomorphism generated by a vector field $\xi^{\mu}$, the generators

$$
H[\xi]=\int_{\Sigma} d^{n-1} x \xi^{\mu} \mathcal{H}_{\mu}
$$


integrated over a Cauchy surface $\Sigma$, have Poisson brackets

$$
\{H[\xi], F\}=\delta_{\xi} F
$$

for any phase space function $F$, where $\delta_{\xi} F$ is the variation of $F$ under the diffeomorphism generated by $\xi$. Further, these generators obey an algebra

$$
\{H[\xi], H[\eta]\}=H[\{\xi, \eta\}]
$$

where $\{\xi, \eta\}$ is the ordinary commutator of vector fields.

The ADM formalism [31] requires some minor modifications: the parameters $\xi^{\mu}$ are replaced by "surface deformation parameters" $\xi^{\perp}$ and $\hat{\xi}^{i}$ [32], given by

$$
\xi^{\perp}=N \xi^{t}, \quad \hat{\xi}^{i}=\xi^{i}+N^{i} \xi^{t}
$$

and the brackets in (4) become the surface deformation brackets

$$
\begin{aligned}
& \{\xi, \eta\}_{S D}^{\perp}=\hat{\xi}^{i} D_{i} \eta^{\perp}-\hat{\eta}^{i} D_{i} \xi^{\perp} \\
& \{\xi, \eta\}_{S D}^{i}=\hat{\xi}^{k} D_{k} \hat{\eta}^{i}-\hat{\eta}^{k} D_{k} \hat{\xi}^{i}+q^{i k}\left(\xi^{\perp} D_{k} \eta^{\perp}-\eta^{\perp} D_{k} \xi^{\perp}\right)
\end{aligned}
$$

where $q_{i j}$ is the spatial metric on $\Sigma$ and $D_{i}$ is the corresponding covariant derivative. On shell, however, the two formalisms are essentially the same [33].

For a manifold with boundary, the story becomes a bit more complicated. As Regge and Teitelboim first noted [34], if $\Sigma$ has a boundary, the generators (2) are not "differentiable" - a variation of the fields gives not only the usual functional derivative of $\mathcal{H}_{\mu}$, but also an added boundary term arising from integration by parts. As a result, the Poisson brackets (3) and (4) acquire new, singular boundary contributions.

While it may be possible to generalize the Poisson algebra to include such terms [35], the simplest remedy is to add a boundary term to the generators,

$$
\bar{H}[\xi]=H[\xi]+B[\xi]=\int_{\Sigma} d^{n-1} x \xi^{\mu} \mathcal{H}_{\mu}+\int_{\partial \Sigma} d^{n-2} x \xi^{\mu} \mathcal{B}_{\mu}
$$

where the boundary term $B[\xi]$ is chosen to cancel the boundary terms coming from the partial integration. The particular form of $B[\xi]$ depends on the choice of boundary conditions, since these determine what is not varied at $\partial \Sigma$. Note that while $H[\xi]$ vanishes on shell, $\bar{H}[\xi]$ need not. It—or, equivalently, the boundary term $B[\xi]$ — may be viewed as a Noether charge associated with the corresponding symmetry [28]. In particular, for a boundary at infinity, suitable choices of $\xi$ give the mass and angular momentum.

In general, the new boundary term may alter the Poisson brackets (4), which become

$$
\{\bar{H}[\xi], \bar{H}[\eta]\}=\bar{H}[\{\xi, \eta\}]+K[\xi, \eta]
$$

where $K[\xi, \eta]$ is a central term. This term was first computed by Brown and Henneaux for $(2+1)$-dimensional asymptotically anti-de Sitter space [20], where it takes a simple form that depends only on Newton's constant and the cosmological constant. A more general form, again computed in the ADM formalism, will be given below. 
An alternative approach the the computation of this central term is based on the covariant canonical formalism [28-30]. This approach starts with the observation that in a well-posed theory, the phase space, considered as the space of initial data, is isomorphic to the space of classical solutions. One can therefore carry out a canonical analysis on this space of solutions, in a covariant manner that does not require a splitting of spacetime into space and time. The covariant canonical derivation of the central term $K[\xi, \eta]$ was first treated in [14]; the results were corrected and generalized in [36,37], and greatly extended in $[38,39]$.

\subsection{Look for a Preferred Subalgebra of Diffeomorphisms of the Circle}

The central term $K[\xi, \eta]$ in the algebra (8) depends strongly on the boundary conditions. Suppose, however, that one can find a subalgebra Diff $S^{1}$ of diffeomorphisms of the circle, that is, a one-parameter subalgebra such that

$$
\{\xi, \eta\}=\xi \eta^{\prime}-\eta \xi^{\prime}
$$

One can then use the general result that the such an algebra has an almost unique central extension [40]: up to redefinitions of the generators,

$$
\{\bar{H}[\xi], \bar{H}[\eta]\}=\bar{H}[\{\xi, \eta\}]+\frac{c}{48 \pi} \int d \varphi\left(\xi^{\prime} \eta^{\prime \prime}-\eta^{\prime} \xi^{\prime \prime}\right)
$$

The algebra (10) is known as a Virasoro algebra; the parameter $c$ is the central charge.

It is at this point that two-dimensionality and conformal symmetry enter the picture. The Virasoro algebra, viewed as the algebra of holomorphic diffeomorphisms of a two-manifold, is the fundamental symmetry algebra of a two-dimensional chiral conformal field theory [41]. Similarly, Diff $S^{1} \times$ Diff $S^{1}$, viewed as an algebra of holomorphic and antiholomorphic diffeomorphisms, is the symmetry algebra of a nonchiral conformal field theory.

The existence of a preferred Diff $S^{1}$ in the higher-dimensional algebra (8) is certainly not guaranteed. But it is also not unreasonable to expect such a subgroup. The BTZ black hole, for instance, is a three-dimensional asymptotically anti-de Sitter spacetime. The boundary of $\mathrm{AdS}_{3}$ is a flat two-dimensional cylinder, so the existence of a pair of Virasoro algebras in the asymptotic symmetry group is no surprise. Similarly, the near-horizon extremal Kerr metric of [22] contains a twisted $\mathrm{AdS}_{3}$ factor, so a Diff $S^{1}$ subgroup is again not too surprising.

For more general black holes, the existence of a Diff $S^{1}$ subgroup is less obvious. But generic black holes do have preferred directions. A stationary black hole in $3+1$ dimensions admits two Killing vectors, for instance, representing time translations and rotations; in the extremal [15] and near-extremal [42] Kerr/CFT correspondences, these pick out the relevant Diff $S^{1}$ factors.

Even more generally, for any "equilibrium" black hole — any black hole characterized by an isolated horizon [43] with a stationary neighborhood — the horizon is a Killing horizon [44]. This means the horizon $\mathcal{H}$ is a null surface generated by a local Killing vector $\chi^{a}$ that becomes null at $\mathcal{H}$. This Killing vector determines a one-dimensional subalgebra of diffeomorphisms for a generic black hole, giving the Diff $S^{1}$ factor used in [14] and [26]. The same Killing vector is important elsewhere in black hole thermodynamics: it fixes the temperature of fields in a black hole background [4], and defines the basic symmetry required for the proof of the generalized second law of thermodynamics [45]. 


\subsection{Use Conformal Field Theory Methods to Extract Physical Information}

The symmetries of a theory give us a powerful tool for extracting physical information. This is particularly true for a two-dimensional conformal field theory, for which the group of symmetries is infinite-dimensional. In fact, the conformal symmetries largely determine such key features as correlation functions [41]. Even more important for our purposes, they determine the asymptotic density of states, that is, the entropy.

Consider a conformal field theory whose holomorphic symmetries are given by the Virasoro algebra (10). As noted earlier, the zero-mode $\bar{H}\left[\xi_{0}\right]$ is a kind of charge, sometimes called the conformal dimension or the conformal weight. Suppose the spectrum of this operator is nonnegative, with a lowest value $\Delta_{0}$. Then the asymptotic density of states at a fixed value $\Delta$ of the weight is [16,17]

$$
\ln \rho \sim 2 \pi \sqrt{\frac{c_{\text {eff }}}{6}\left(\Delta-\frac{c}{24}\right)} \quad \text { with } c_{\text {eff }}=c-24 \Delta_{0}
$$

This remarkable result, known as the Cardy formula, determines the microcanonical entropy in terms of a few parameters that characterize the symmetry, independent of any other details of the theory. Logarithmic [46] and higher order [47,48] corrections are known as well. The derivation of the Cardy formula is fairly straightforward—see, for instance [49]—but it involves a duality between high- and low-energy states; I think it is fair to say that we do not yet have a very deep physical understanding of the result.

The Cardy formula comes in a canonical version as well. Instead of fixing the weight $\Delta$, let us fix the temperature $T$. Then (see, for instance, section 8 of [50])

$$
S \sim \frac{\pi^{2}}{3} c T
$$

There is a slightly subtle point here: temperature is normally associated with periodicity in imaginary time, but here the relevant periodicity is that of the $S^{1}$ picked out by the Virasoro algebra. We will see examples of this in Section 4.

Going beyond the entropy, one can also use conformal field theory techniques to explore such matters as greybody factors, superradiance, and the Hawking temperature (see, for example [51-53]). For the most part, however, such work lies outside the scope of this review.

\section{Central Terms in the ADM Formalism}

The discussion in the preceding section was somewhat abstract, and it is useful to look at some specific examples. To do so, I must first fill in a few more details. I will work in the ADM approach, which is perhaps less elegant than the covariant phase space method, but also requires less formalism.

In $n$ spacetime dimensions, the ADM form of a general metric is

$$
d s^{2}=-N^{2} d t^{2}+q_{i j}\left(d x^{i}+N^{i} d t\right)\left(d x^{j}+N^{j} d t\right), \quad(i=1,2, \ldots, n-1)
$$

The momentum conjugate to the spatial metric $q_{i j}$ is

$$
\pi^{i j}=\sqrt{q}\left(K^{i j}-q^{i j} K\right)
$$


where $K_{i j}$ is the extrinsic curvature of a slice of constant $t$. I denote the spatial covariant derivative compatible with $q_{i j}$ by $D_{i}$, and lower and raise indices with $q_{i j}$ and its inverse $q^{i j}$.

The constraints are generated by the Hamiltonian

$$
H\left[\xi^{\perp}, \hat{\xi}^{i}\right]=\int_{\Sigma} d^{n-1} x\left(\xi^{\perp} \mathcal{H}+\hat{\xi}^{i} \mathcal{H}_{i}\right)
$$

where

$$
\mathcal{H}=\frac{1}{\sqrt{q}}\left(\pi^{i j} \pi_{i j}-\frac{1}{n-2} \pi^{2}\right)-\sqrt{q}^{(n-1)} R, \quad \mathcal{H}^{i}=-2 D_{j} \pi^{i j}
$$

Here, ${ }^{(n-1)} R$ is the spatial curvature scalar, and the integral is over a Cauchy surface $\Sigma$ [54]. The transformation generated by (15) is not a diffeomorphism, but is equivalent on shell to the diffeomorphism generated by the vector field $\xi^{\mu}$ given in (5).

On a manifold without a spatial boundary, the generators have Poisson brackets

$$
\{H[\xi], H[\eta]\}=H\left[\{\xi, \eta\}_{S D}\right]
$$

where the surface deformation brackets $\{\cdot, \cdot\}_{S D}$ are given by (6). As noted above, for a manifold with boundary one must add boundary terms to make the variation of the constraints well-defined. The resulting modification of the Poisson algebra (17) depends on the exact choice of boundary conditions.

But even without these details, one can learn a good deal. The key observation is that, by construction, the new generator $\bar{H}[\xi]=H[\xi]+B[\xi]$ has a variation with no boundary contributions. Their Poisson bracket is thus simply the standard one,

$$
\{\bar{H}[\xi], \bar{H}[\eta]\}=\int d^{n-1} x\left(\frac{\delta \bar{H}[\xi]}{\delta q_{i j}} \frac{\delta \bar{H}[\eta]}{\delta \pi^{i j}}-\frac{\delta \bar{H}[\eta]}{\delta q_{i j}} \frac{\delta \bar{H}[\xi]}{\delta \pi^{i j}}\right)
$$

From (8), the central term is thus

$$
\begin{aligned}
K[\xi, \eta] & =\{\bar{H}[\xi], \bar{H}[\eta]\}-\bar{H}\left[\{\xi, \eta\}_{S D}\right] \\
& =\int d^{n-1} x\left(\frac{\delta \bar{H}[\xi]}{\delta q_{i j}} \frac{\delta \bar{H}[\eta]}{\delta \pi^{i j}}-\frac{\delta \bar{H}[\eta]}{\delta q_{i j}} \frac{\delta \bar{H}[\xi]}{\delta \pi^{i j}}\right)-H\left[\{\xi, \eta\}_{S D}\right]-B\left[\{\xi, \eta\}_{S D}\right]
\end{aligned}
$$

The variational derivatives in (19) can be rearranged to give $H\left[\{\xi, \eta\}_{S D}\right]$, but only after integration by parts. A tedious but straightforward calculation yields

$$
\begin{aligned}
K[\xi, \eta]=-B\left[\{\xi, \eta\}_{S D}\right] & \\
-\frac{1}{8 \pi G} \int_{\partial \Sigma} d^{n-2} x \sqrt{\sigma} n^{k}[ & -\frac{1}{2} \frac{1}{\sqrt{q}}\left(\hat{\xi}_{k} \eta^{\perp}-\hat{\eta}_{k} \xi^{\perp}\right) \mathcal{H}-\left(\hat{\xi}_{i} \eta^{\perp}-\hat{\eta}_{i} \xi^{\perp}\right)^{(n-1)} R_{k}^{i} \\
& +\left(D_{i} \hat{\xi}_{k} D^{i} \eta^{\perp}-D_{i} \hat{\eta}_{k} D^{i} \xi^{\perp}\right)-\left(D_{i} \hat{\xi}^{i} D_{k} \eta^{\perp}-D_{i} \hat{\eta}^{i} D_{k} \xi^{\perp}\right) \\
& \left.+\frac{1}{\sqrt{q}}\left(\hat{\eta}_{k} \pi^{m n} D_{m} \hat{\xi}_{n}-\hat{\xi}_{k} \pi^{m n} D_{m} \hat{\eta}_{n}\right)+\frac{1}{\sqrt{q}} \pi_{i k}\{\xi, \eta\}_{S D}^{i}\right]
\end{aligned}
$$

where $\sigma_{i j}$ is the induced metric on $\partial \Sigma$ and $n^{k}$ is the unit normal to $\partial \Sigma$.

The central term (20) depends on the boundary contribution $B\left[\{\xi, \eta\}_{S D}\right]$. But for the computation of the central charge of a Virasoro subalgebra, we do not need to know this term (although we ought to 
check that it is finite). Indeed, the boundary term depends on the deformation parameters only in the combination $\{\xi, \eta\}=\xi \eta^{\prime}-\eta \xi^{\prime}$. The central term in a Virasoro algebra (10), in contrast, depends on the combination $\xi^{\prime} \eta^{\prime \prime}-\eta^{\prime} \xi^{\prime \prime}$, which cannot be built from $\{\xi, \eta\}$ and its derivatives. If such a structure appears in (20), it is thus necessarily a genuine central term.

To compute black hole entropy from the canonical version (12) of the Cardy formula, it is sufficient to know the central charge and the temperature. To use the microcanonical form (11), on the other hand, one needs the conformal weight, which is obtained from the boundary term $B[\xi]$. This term does not have a simple, universal form - it depends on the choice of boundary conditions-but it must be chosen to cancel the boundary variation of $H[\xi]$. This variation is [55]

$$
\begin{aligned}
\delta H[\xi]=\cdots-\frac{1}{16 \pi G} \int_{\partial \Sigma} d^{n-2} x\{\sqrt{\sigma}[ & \xi^{\perp}\left(n^{k} \sigma^{\ell m}-n^{m} \sigma^{\ell k}\right) D_{m} \delta q_{k \ell} \\
& \left.\left.-D_{m} \xi^{\perp}\left(n^{k} \sigma^{\ell m}-n^{m} \sigma^{\ell k}\right) \delta q_{k \ell}\right]+2 \hat{\xi}^{i} \delta \pi^{n}{ }_{i}-\hat{\xi}^{n} \pi^{i j} \delta q_{i j}\right\}
\end{aligned}
$$

Note that this variation determines the boundary term only up to an additive constant, which must be fixed by the physics and can sometimes be troublesome.

\section{Some Examples}

With these general results in hand, we can now look at a few concrete examples.

\subsection{The BTZ Black Hole}

The BTZ metric [6] describes a (2+1)-dimensional asymptotically anti-de Sitter black hole. It is a stationary, axisymmetric solution of the vacuum Einstein field equations with a cosmological constant $\Lambda=-1 / \ell^{2}$, and is given in ADM form by

$$
d s^{2}=N^{2} d t^{2}-N^{-2} d r^{2}-r^{2}\left(d \varphi+N^{\varphi} d t\right)^{2}
$$

with

$$
N=\left(-8 G M+\frac{r^{2}}{\ell^{2}}+\frac{16 G^{2} J^{2}}{r^{2}}\right)^{1 / 2}, \quad N^{\varphi}=-\frac{4 G J}{r^{2}} \quad(|J| \leq M \ell)
$$

The only nonvanishing component of the ADM momentum is

$$
\pi^{r \varphi}=\frac{r_{+} r_{-}}{\ell r^{2}}
$$

Pure anti-de Sitter space is described by a similar metric, with $J=0$ and $8 G M=-1$.

Like every solution of the vacuum field equations in $2+1$ dimensions, the BTZ black hole has constant curvature. Nevertheless, somewhat surprisingly, it is a true black hole. In particular, it has an event horizon at $r_{+}$and, when $J \neq 0$, an inner Cauchy horizon at $r_{-}$, where

$$
r_{ \pm}^{2}=4 G M \ell^{2}\left(1 \pm\left[1-\left(\frac{J}{M \ell}\right)^{2}\right]^{1 / 2}\right), \quad \text { i.e., } \quad M=\frac{r_{+}^{2}+r_{-}^{2}}{8 G \ell^{2}}, \quad J=\frac{r_{+} r_{-}}{4 G \ell}
$$


The horizon rotates at an angular velocity

$$
\Omega_{H}=-N^{\varphi}\left(r_{+}\right)=\frac{r_{-}}{r_{+} \ell}
$$

Kruskal coordinates were derived in [56]; the Penrose diagram is essentially the same as that of an asymptotically anti-de Sitter black hole in $3+1$ dimensions.

In 1986, Brown and Henneaux stumbled across an aspect of what we would now call the AdS/CFT correspondence [20,57]. Analyzing the asymptotic symmetries of general relativity in $(2+1)$-dimensional asymptotically anti-de Sitter space, they found a pair of commuting Virasoro algebras - the symmetry group of a two-dimensional conformal field theory-with central charges

$$
c^{ \pm}=\frac{3 \ell}{2 G}
$$

To obtain this result, Brown and Henneaux imposed boundary conditions

$$
g_{a b} \sim\left(\begin{array}{ccc}
-\frac{r^{2}}{\ell^{2}}+\mathcal{O}(1) & \mathcal{O}\left(\frac{1}{r^{3}}\right) & \mathcal{O}(1) \\
& \frac{\ell^{2}}{r^{2}}+\mathcal{O}\left(\frac{1}{r^{4}}\right) & \mathcal{O}\left(\frac{1}{r^{3}}\right) \\
& & r^{2}+\mathcal{O}(1)
\end{array}\right)
$$

at spatial infinity. (My index order is $(t, r, \varphi)$.) These conditions are satisfied by the BTZ black hole, as well as by pure anti-de Sitter space and anti-de Sitter space with point particles. It is straightforward to check that the diffeomorphisms that respect these boundary conditions are generated by two families of vector fields $\xi^{ \pm}$, with

$$
\begin{aligned}
\xi^{ \pm t} & =\frac{\ell}{2}\left(T^{ \pm}+\frac{\ell^{2}}{2 r^{2}} \partial_{\varphi}^{2} T^{ \pm}\right) \\
\xi^{ \pm r} & =\mp \frac{r}{2} \partial_{\varphi} T^{ \pm} \\
\xi^{ \pm \varphi} & = \pm \frac{1}{2}\left(T^{ \pm}-\frac{\ell^{2}}{2 r^{2}} \partial_{\varphi}^{2} T^{ \pm}\right)
\end{aligned}
$$

where $T^{ \pm}=T^{ \pm}(\varphi \pm t / \ell)$ are left- and right-moving modes on the asymptotic boundary.

The vector fields (29) are normalized so that

$$
\begin{aligned}
& \left\{\xi^{ \pm}\left[T_{1}^{ \pm}\right], \xi^{ \pm}\left[T_{2}^{ \pm}\right]\right\}=\xi^{ \pm}\left[T_{1}^{ \pm} \partial_{\varphi} T_{2}^{ \pm}-T_{2}^{ \pm} \partial_{\varphi} T_{1}^{ \pm}\right] \\
& \left\{\xi^{+}\left[T_{1}^{+}\right], \xi^{-}\left[T_{2}^{-}\right]\right\}=0
\end{aligned}
$$

From (9) and (10), the corresponding generators of surface deformations should form a pair of commuting Virasoro algebras. To find the central charges, we can use the general result (20). The relevant three-derivative terms are

$$
\begin{aligned}
K[\xi, \eta]=\cdots-\frac{1}{8 \pi G} \int_{\partial \Sigma} d \varphi r N & {\left[-\left(\hat{\xi}_{r} \eta^{\perp}-\hat{\eta}_{r} \xi^{\perp}\right)^{(2)} R_{r}^{r}\right.} \\
& \left.+\left(D_{\varphi} \hat{\xi}_{r} D^{\varphi} \eta^{\perp}-D_{\varphi} \hat{\eta}_{r} D^{\varphi} \xi^{\perp}\right)-\left(D_{\varphi} \hat{\xi}^{\varphi} D_{r} \eta^{\perp}-D_{\varphi} \hat{\eta}^{\varphi} D_{r} \xi^{\perp}\right)\right]
\end{aligned}
$$


where $D_{\varphi} \hat{\xi}^{r}=\partial_{\varphi} \hat{\xi}^{r}-r N^{2} \hat{\xi}^{\varphi}$ and $D_{\varphi} \hat{\xi}^{\varphi}=\partial_{\varphi} \hat{\xi}^{\varphi}+\frac{1}{r} \hat{\xi}^{r}$ are spatial covariant derivatives. A straightforward calculation yields

$$
K\left[\xi^{ \pm}\left[T_{1}^{ \pm}\right], \xi^{ \pm}\left[T_{2}^{ \pm}\right]\right]=\cdots+\frac{\ell}{32 \pi G} \int_{\partial \Sigma} d \varphi\left(\partial_{\varphi} T_{1}^{ \pm} \partial_{\varphi}^{2} T_{2}^{ \pm}-\partial_{\varphi} T_{2}^{ \pm} \partial_{\varphi}^{2} T_{1}^{ \pm}\right)
$$

Comparing this to the general Virasoro algebra (10), we can read off the central charges, confirming the results (27).

To use the microcanonical Cardy formula, we also need the boundary term $B[\xi]$. This was also computed by Brown and Henneaux. From the boundary conditions (28), it is easy to see that the only nonvanishing terms in the variation (22) are

$$
\begin{aligned}
\delta H[\xi] & =\cdots-\frac{1}{16 \pi G} \int d \varphi\left\{r n^{r} \sigma^{\varphi \varphi}\left[\xi^{\perp}\left(D_{\varphi} \delta q_{r \varphi}-D_{r} \delta q_{\varphi \varphi}\right]+r n^{r} \sigma^{\varphi \varphi} D_{r} \xi^{\perp} \delta q_{\varphi \varphi}+2 \hat{\xi}^{\varphi} \delta \pi^{r}{ }_{\varphi}\right\}\right. \\
& =\cdots-\frac{1}{16 \pi G} \int d \varphi\left[\xi^{t} \delta\left(\frac{r_{+}^{2}+r_{-}^{2}}{\ell^{2}}\right)+\hat{\xi}^{\varphi} \delta\left(\frac{2 r_{+} r_{-}}{\ell}\right)\right]=\cdots-\frac{1}{2 \pi} \delta \int d \varphi\left[\xi^{t} M+\xi^{\varphi} J\right]
\end{aligned}
$$

justifying the notation $M$ and $J$ for the mass and angular momentum of the black hole. Evaluated on the asymptotic symmetries (29), the boundary term is then

$$
B\left[T^{ \pm}\right]=\frac{\left(r_{+} \pm r_{-}\right)^{2}}{32 \pi G \ell} \int d \varphi T^{ \pm}+B_{0}
$$

In particular, for the zero modes $T_{0}^{ \pm}=1$, we have

$$
\Delta^{ \pm}=B\left[T_{0}^{ \pm}\right]=\frac{\left(r_{+} \pm r_{-}\right)^{2}}{16 G \ell}+\frac{\ell}{16 G}
$$

where the constant $B_{0}$ has been fixed by demanding that anti-de Sitter space have $\Delta^{ \pm}=0$. As Strominger [7] and Birmingham, Sachs, and Sen [8] observed, these conformal weights and the central charges (27) then determine an entropy

$$
S=2 \pi \sqrt{\frac{c^{+}}{6}\left(\Delta^{+}-\frac{c^{+}}{24}\right)}+2 \pi \sqrt{\frac{c^{-}}{6}\left(\Delta^{-}-\frac{c^{-}}{24}\right)}=\frac{2 \pi r_{+}}{4 G}
$$

one quarter of the horizon "area" $2 \pi r_{+}$, matching the standard Bekenstein-Hawking expression.

It is interesting to consider the canonical version (12) of the Cardy formula as well. The BTZ black hole has a Hawking temperature

$$
T_{H}=\frac{\kappa}{2 \pi}=\frac{r_{+}^{2}-r_{-}^{2}}{2 \pi \ell r_{+}}
$$

The inverse of this temperature gives the periodicity of Greens functions in imaginary time. For our two Virasoro algebras, though, the relevant periodicities are in the coordinates $t^{ \pm}=t \pm \ell \varphi$ that appear in the asymptotic diffeomorphisms. To obtain the corresponding "left" and "right" temperatures, following [15], we first note that the modes of a scalar field in a BTZ background take the form

$$
\phi \sim F(r) e^{i m \varphi-i \omega t}=F(r) e^{-i \omega^{+} t^{+}-i \omega^{-} t^{-}} \quad \text { with } \quad \omega^{ \pm}=\frac{1}{2}\left(\omega \mp \frac{m}{\ell}\right)
$$


The standard Boltzmann factor depends on both the energy and the angular velocity $\Omega_{H}$, and can be written as

$$
e^{-\beta_{H}\left(\omega-m \Omega_{H}\right)}=e^{-\beta^{+} \omega^{+}-\beta^{-} \omega^{-}} \quad \text { with } \quad \beta^{ \pm}=\beta\left(1 \pm \Omega_{H} \ell\right)=\frac{2 \pi \ell}{r_{+} \mp r_{-}}
$$

Substituting the corresponding temperatures $T^{ \pm}=1 / \beta^{ \pm}$into the canonical Cardy formula, we obtain

$$
S=\frac{\pi^{2}}{3} c^{+} T^{+}+\frac{\pi^{2}}{3} c^{-} T^{-}=\frac{2 \pi r_{+}}{4 G}
$$

again giving the standard Bekenstein-Hawking entropy.

In some ways, the BTZ black hole is a very special case: the asymptotic boundary is a two-dimensional cylinder, so an asymptotic conformal field theory seems quite natural. But the result also has a simple extension. Many near-extremal black holes have a near-horizon geometry of the form $B T Z \times$ trivial, and the results of this section can be applied almost unchanged, except for appropriate redefinitions of constants. For a good review, see [21].

\subsection{The Extremal Kerr Black Hole}

As our next example, let us consider the near-horizon geometry of the extremal Kerr black hole $[15,58]$, a system that has attracted a good deal of recent attention. In Boyer-Lindquist coordinates, the ADM form of the extremal Kerr metric is [59]

$$
d s^{2}=-N^{2} d t^{2}+\frac{\Sigma}{\Delta} d r^{2}+\frac{A \sin ^{2} \theta}{\Sigma}\left(d \varphi+N^{\varphi} d t\right)^{2}+\Sigma d \theta^{2}
$$

where

$$
\begin{aligned}
& \Delta=\left(r-r_{+}\right)^{2}, \quad \Sigma=r^{2}+r_{+}{ }^{2} \cos ^{2} \theta, \quad A=\left(r^{2}+r_{+}{ }^{2}\right)^{2}-\left(r-r_{+}\right)^{2} r_{+}{ }^{2} \sin ^{2} \theta \\
& N=\sqrt{\frac{\Sigma \Delta}{A}}, \quad N^{\varphi}=\frac{2 r_{+}{ }^{2} r}{A}
\end{aligned}
$$

This black hole has a mass $G M=r_{+}$, an angular momentum $J=r_{+}^{2} / G$, and a horizon at $r=r_{+}$ that rotates with an angular velocity

$$
\Omega_{H}=N^{\varphi}\left(r_{+}\right)=\frac{1}{2 r_{+}}
$$

At extremality, the Kerr black hole has an infinite throat: that is, the proper distance from the horizon $r_{+}$to any point $r>r_{+}$is infinite. To focus in on the near-horizon region, Bardeen and Horowitz considered a coordinate transformation [22]

$$
\bar{t}=\frac{\lambda t}{2 m}, \quad y=\frac{\lambda m}{r-m}, \quad \bar{\varphi}=\varphi-\Omega_{H} t
$$

followed by a limit $\lambda \rightarrow 0$. The resulting near-horizon extremal Kerr, or NHEK, metric is

$$
d s^{2}=r_{+}^{2}\left(1+\cos ^{2} \theta\right)\left(\frac{-d \bar{t}^{2}+d y^{2}}{y^{2}}+\Lambda^{2}\left(d \bar{\varphi}+\frac{d \bar{t}}{y}\right)^{2}+d \theta^{2}\right)
$$


with

$$
\Lambda=\frac{2 \sin \theta}{1+\cos ^{2} \theta}
$$

The only nonvanishing component of the canonical momentum is

$$
\pi^{y \bar{\varphi}}=\frac{\sqrt{q}}{2 N} q^{y y} \partial_{y} N^{\bar{\varphi}}=-\frac{\Lambda}{2}
$$

Guica et al. consider boundary conditions [15]

$$
\delta g_{a b} \sim\left(\begin{array}{cccc}
\mathcal{O}\left(\frac{1}{y^{2}}\right) & \mathcal{O}(1) & \mathcal{O}(y) & \mathcal{O}(1) \\
& \mathcal{O}\left(\frac{1}{y}\right) & \mathcal{O}(1) & \mathcal{O}\left(\frac{1}{y}\right) \\
& & \mathcal{O}(y) & \mathcal{O}(y) \\
& & & \mathcal{O}(1)
\end{array}\right)
$$

near the asymptotic boundary $y=0$. (My index order is $(\bar{t}, y, \theta, \bar{\varphi})$.) These conditions, which are strong enough to lead to finite charges, are preserved by the diffeomorphisms

$$
\xi^{\bar{\varphi}}=\epsilon(\bar{\varphi}), \quad \xi^{y}=y \partial_{\bar{\varphi}} \epsilon(\bar{\varphi})
$$

which satisfy a Diff $S^{1}$ algebra. (Constant time translations are also permitted, but are not relevant to the dual conformal field theory.) Since (49) includes no $\xi^{\perp}$ component, the sole contributions to the central term (20) come from the terms involving the canonical momentum $\pi^{i j}$. The only three-derivative term is

$$
\begin{aligned}
& K[\xi, \eta]=\cdots-\frac{1}{8 \pi G} \int d^{2} x\left(\hat{\eta}^{y} \pi^{m n} D_{m} \hat{\xi}_{n}-\hat{\xi}^{y} \pi^{m n} D_{m} \hat{\eta}_{n}\right) \\
& =\cdots+\frac{1}{8 \pi G} \int d \theta d \bar{\varphi} \frac{\Lambda}{2}\left(\eta^{y} q_{y y} \partial_{\bar{\varphi}} \xi^{y}-\xi^{y} q_{y y} \partial_{\bar{\varphi}} \eta^{y}\right) \\
& =\cdots-\frac{r_{+}^{2}}{16 \pi G} \int d \theta \Lambda\left(1+\cos ^{2} \theta\right) \int d \bar{\varphi}\left(\partial_{\bar{\varphi}} \eta^{\bar{\varphi}} \partial_{\bar{\varphi}}^{2} \xi^{\bar{\varphi}}-\partial_{\bar{\varphi}} \xi^{\bar{\varphi}} \partial_{\bar{\varphi}}^{2} \eta^{\bar{\varphi}}\right) \\
& =\cdots+\frac{r_{+}^{2}}{4 \pi G} \int d \bar{\varphi}\left(\partial_{\bar{\varphi}} \xi^{\bar{\varphi}} \partial_{\bar{\varphi}}^{2} \eta^{\bar{\varphi}}-\partial_{\bar{\varphi}} \eta^{\bar{\varphi}} \partial_{\bar{\varphi}}^{2} \xi^{\bar{\varphi}}\right)
\end{aligned}
$$

Comparing to (10), we see that we one again have a Virasoro algebra, with central charge

$$
c=\frac{12 r_{+}^{2}}{G}=12 J
$$

There is an important difference between this central charge and that of the BTZ black hole. For the BTZ black hole, $c$ depends only on the cosmological constant, and not on the particular parameters of the black hole. It is thus plausible to conjecture that a single conformal field theory encompasses all $(2+1)$-dimensional black holes. Here, in contrast, $c$ depends on $J$, implying a different dual conformal field theory for each value of angular momentum.

This may not be important for extremal rotating black holes, which have a vanishing temperature and do not undergo Hawking radiation. But let us suppose — as suggested, for instance, in [60]— that a single conformal field theory also describes non-extremal black holes with the same angular momentum. Then Hawking radiation, which will typically change the value of $J$, will take us from one conformal dual 
to another. This suggests that a full treatment of Hawking radiation, including its back-reaction on the black hole, may involve flows between conformal field theories.

To use the microcanonical Cardy formula, we need the boundary term $B[\xi]$. Again, the absence of a $\xi^{\perp}$ component in the deformation (49) makes this easy to compute: the only relevant term in (21) is

$$
\begin{aligned}
\delta H[\xi] & =\cdots-\frac{1}{16 \pi G} \int_{\partial \Sigma} d \theta d \bar{\varphi}\left(2 \hat{\xi}^{\bar{\varphi}} \delta \pi^{y} \bar{\varphi}\right) \\
& =\cdots-\frac{1}{16 \pi G} \int d \bar{\varphi} \epsilon(\bar{\varphi}) \int d \theta\left(-\frac{\Lambda}{2} \cdot r_{+}^{2}\left(1+\cos ^{2} \theta\right) \Lambda^{2}\right) \\
& =\cdots+\frac{r_{+}^{2}}{2 \pi G} \int d \bar{\varphi} \epsilon(\bar{\varphi}) \int d \theta \frac{\sin ^{3} \theta}{\left(1+\cos ^{2} \theta\right)^{2}}=\cdots+\frac{r_{+}^{2}}{G} \int \frac{d \bar{\varphi}}{2 \pi} \epsilon(\bar{\varphi})
\end{aligned}
$$

If we now take the undetermined constant $B_{0}$ in the boundary term to be zero-a plausible assumption, but not one that is obviously true - the Cardy formula yields

$$
S=2 \pi \sqrt{\frac{c}{6}\left(\Delta-\frac{c}{24}\right)}=\frac{2 \pi r_{+}^{2}}{G},
$$

which is the correct Bekenstein-Hawking entropy for the extremal Kerr black hole.

We can also use the canonical version of the Cardy formula. This is a bit subtle: the Hawking temperature for a general Kerr black hole is

$$
T=\frac{r_{+}-M}{4 \pi M r_{+}}
$$

which vanishes for an extremal black hole. Following Guica et al. [15], though, we can obtain an appropriate temperature as a limit. For a nonextremal black hole, the modes of the Frolov-Thorne vacuum [61] are of the form

$$
\phi \sim F(r, \theta) e^{i m \varphi-i \omega t} \sim \bar{F}(y, \theta) e^{i n_{L} \bar{\varphi}-i n_{R} \bar{t}} \quad \text { with } \quad n_{L}=m, n_{R}=\frac{1}{\lambda}(2 M \omega-m)
$$

where I have performed the coordinate transformation (44) but not yet taken the $\lambda \rightarrow 0$ limit. As in the BTZ case, we can compute the corresponding Boltzmann factor,

$$
e^{-\beta_{H}\left(\omega-m \Omega_{H}\right)}=e^{-\beta_{R} n_{R}-\beta_{L} n_{L}} \quad \text { with } \quad \beta_{R}=2 \pi\left(\frac{r_{+}-2 \Omega_{H} r_{+} M}{r_{+}-M}\right), \quad \beta_{L}=\frac{2 \pi \lambda r_{+}}{r_{+}-M} .
$$

In the extremal limit, $r_{+} \rightarrow M$, the right temperature remains finite,

$$
T_{R}=1 / \beta_{R} \rightarrow \frac{1}{2 \pi}
$$

The canonical Cardy formula (12) then gives

$$
S=\frac{\pi^{2}}{3} c T=\frac{2 \pi r_{+}^{2}}{G}
$$

again matching the Bekenstein-Hawking entropy.

A similar analysis has been performed for other extremal black holes (see, for example, $[62,63]$ ), and has been repeated for the extremal Kerr solution without taking the near-horizon limit [26]. But although some attempts have been made to extend the method to non-extremal black holes [42,64], the situation remains murky. So for the non-extremal case, it is worthwhile to look for an alternative approach. 


\subsection{A Generic $(3+1)$-Dimensional Black Hole}

In the preceding examples, we looked at asymptotic boundaries far from the black hole horizon: at infinity for the BTZ black hole, and at the "infinity" of the near-horizon region for the extremal Kerr black hole. But as noted in Section 2, it is also natural to look at boundary conditions at the horizon itself.

Consider an arbitrary stationary black hole in $3+1$ dimensions [26]. Such a black hole is necessarily axisymmetric [65]: that is, it admits two Killing vectors $T^{a}$ and $\Phi^{a}$ that generate time translations and rotations. For the near-horizon metric, we can write a general ADM form

$$
d s^{2}=-N^{2} d t^{2}+d \rho^{2}+q_{\varphi \varphi}\left(d \varphi+N^{\varphi} d t\right)^{2}+q_{z z} d z^{2}
$$

where $\rho$ is the proper distance from the horizon and $z$ is an angular coordinate such as $\cos \theta$. Even before imposing any field equations, it can be shown that the requirement of finite curvature at the horizon sharply restricts the behavior of the metric: it must have a near-horizon expansion [66]

$$
\begin{array}{ll}
N=\kappa_{H} \rho+\frac{1}{3 !} \kappa_{2}(z) \rho^{3}+\ldots & q_{\varphi \varphi}=\left[q_{H}\right]_{\varphi \varphi}(z)+\frac{1}{2}\left[q_{2}\right]_{\varphi \varphi}(z) \rho^{2}+\ldots \\
N^{\varphi}=-\Omega_{H}-\frac{1}{2} \omega_{2}(z) \rho^{2}+\ldots & q_{z z}=\left[q_{H}\right]_{z z}(z)+\frac{1}{2}\left[q_{2}\right]_{z z}(z)\left(\rho^{2}\right)+\ldots
\end{array}
$$

where the surface gravity $\kappa_{H}$ and the horizon angular velocity $\Omega_{H}$ are constants. The only nonzero component of the canonical momentum $\pi^{i j}$ near the horizon is then

$$
\pi^{\rho \varphi}=-\frac{\omega_{2}}{2 \kappa_{H}} \sqrt{q}+\mathcal{O}\left(\rho^{2}\right)
$$

Now, for a stationary black hole_or, more generally, a black hole with a stationary neighborhood around an isolated horizon [44]—the horizon $\mathcal{H}$ is a Killing horizon. This means there exists a Killing vector

$$
\chi^{a}=T^{a}+\Omega_{H} \Phi^{a}
$$

that is null at $\mathcal{H}$ and is normal to $\mathcal{H}$. Ideally, this is the place we would impose boundary conditions. As noted earlier, though, this has so far proven very difficult. We can, however, "stretch" the horizon into a timelike boundary $\mathcal{H}_{s}$ just outside the true horizon, determine a conformal dual there, and then take the limit as $\mathcal{H}_{s}$ approaches $\mathcal{H}$.

In particular, let us "stretch" the Killing vector, by requiring that a new Killing vector

$$
\bar{\chi}^{a}=T^{a}+\bar{\Omega} \Phi^{a}
$$

be null at the intersection of the stretched horizon with our Cauchy surface $\Sigma$. For the metric (59), this is the requirement that

$$
\bar{\chi}^{2}=0=-N^{2}+q_{\varphi \varphi}\left(N^{\varphi}+\bar{\Omega}\right)^{2}=-N^{2}+q_{\varphi \varphi}\left(\Omega_{H}-\bar{\Omega}\right)^{2}+\mathcal{O}\left(\rho^{3}\right)
$$

The parameter

$$
\bar{\varepsilon}=\Omega_{H}-\bar{\Omega}
$$


which measures the "stretching" of the Killing vector $\bar{\chi}^{a}$, is of order $\rho$-essentially the proper distance from the stretched horizon to the true horizon — as is standard in the membrane paradigm [25].

At the stretched horizon $\mathcal{H}_{s}$, we can independently specify the surface deformation parameters $\left(\xi^{\perp}, \hat{\xi}^{i}\right)$ and their normal derivatives. The transformations that leave the metric (59) fixed at the boundary are then

$$
\begin{aligned}
& \delta_{\xi} N=0 \Rightarrow \hat{\xi}^{\rho}=-\bar{\varepsilon} \rho \partial_{\varphi} \xi^{t}=-\rho \bar{\partial}_{t} \xi^{t} \\
& \delta_{\xi} N^{\rho}=0 \Rightarrow \rho \partial_{\rho} \xi^{t}=-\frac{\bar{\varepsilon}^{2}}{\kappa_{H}^{2}} \partial_{\varphi}^{2} \xi^{t}=-\frac{1}{\kappa_{H}^{2}} \bar{\partial}_{t}^{2} \xi^{t} \\
& \delta_{\xi} N^{\varphi}=0 \Rightarrow \hat{\xi}^{\varphi}=\frac{\kappa_{H}^{2} \rho^{2}}{\bar{\varepsilon}} q^{\varphi \varphi} \xi^{t}=\bar{\varepsilon} \xi^{t} \\
& \delta q_{\rho \varphi}=0 \Rightarrow \rho \partial_{\rho} \hat{\xi}^{\varphi}=\bar{\varepsilon} \rho^{2} q^{\varphi \varphi} \partial_{\varphi}^{2} \xi^{t}-\omega_{2} \rho^{2} \xi^{t}=\frac{\bar{\varepsilon}}{\kappa_{H}^{2}} \bar{\partial}_{t}^{2} \xi^{t}-\omega_{2} \rho^{2} \xi^{t}
\end{aligned}
$$

where $\xi^{t}=\xi^{t}(\varphi-\bar{\Omega} t)$ and

$$
\bar{\partial}_{t}=\partial_{t}-N^{i} \partial_{i} \sim \partial_{t}+\Omega_{H} \partial_{\varphi}
$$

is a convective derivative.

In our earlier examples, the surface deformations were functions of an angular coordinate, and there was a natural choice of periodicity of the modes. Here, the relevant variable is really an affine parameter along the Killing vector, and the choice is not unique. A particularly nice choice of modes is

$$
\xi_{n}^{t}=\frac{1}{2} e^{i n(\varphi-\bar{\Omega} t) / \bar{\varepsilon}}
$$

which are chosen in order that $\bar{\partial}_{t} \tilde{\xi}_{n}^{t}=i n \tilde{\xi}_{n}^{t}$. The resulting frequencies blow up at the horizon, but this is the ordinary blue shift as seen by an outside observer: a corotating observer with four-velocity $u^{a}=\left(T^{a}-N^{\varphi} \Phi^{a}\right) / N$ will see a frequency $k_{a} u^{a}=n / N(1+\mathcal{O}(\rho))$, the usual blue shift factor. Other possible modes are discussed in [26]; they typically lead to different, and sometimes divergent, central charges and conformal weights, but to the same entropy.

The normalization of (68) has been chosen to ensure that the surface deformation brackets (6) of these modes are consistent, with [67]

$$
\left\{\xi_{m}, \xi_{n}\right\}_{S D}^{t}=i(m-n) \xi_{m+n}^{t}
$$

We thus expect a Virasoro algebra to once again be present. The relevant contributions to the central term (20) are now

$$
\begin{aligned}
K[\xi, \eta] & =-\frac{1}{8 \pi G} \int_{\partial \Sigma} d^{2} x \sqrt{\sigma} n^{k}\left[\left(D_{i} \hat{\xi}_{k} D^{i} \eta^{\perp}-D_{i} \hat{\xi}^{i} D_{k} \eta^{\perp}\right)-(\xi \leftrightarrow \eta)\right] \\
& =-\frac{1}{4 \pi G \kappa_{H}} \frac{\mathcal{A}}{2 \pi} \int d \varphi\left[\bar{\partial}_{t} \xi^{t} \bar{\partial}_{t}^{2} \eta^{t}-\bar{\partial}_{t} \eta^{t} \bar{\partial}_{t}^{2} \xi^{t}\right]
\end{aligned}
$$

where $\mathcal{A}$ is the horizon area. Here I have used (66) for both the components of $\xi$ and $\eta$ and their radial derivatives, along with the identity

$$
\int d^{2} x \sqrt{\sigma} F(\varphi)=\frac{\mathcal{A}}{2 \pi} \int d \varphi F(\varphi)
$$


which follows from the fact that the boundary metric $\sigma_{i j}$ is independent of $\varphi$. The expression (70) may again be recognized as the central term of a Virasoro algebra, with central charge

$$
c=\frac{3 \mathcal{A}}{2 \pi G \kappa_{H}}
$$

It is now easy to use the canonical form of the Cardy formula. The Hawking temperature of a black hole with metric (59) is

$$
T_{H}=\frac{\kappa_{H}}{2 \pi}
$$

The coordinate along our stretched horizon is $(\varphi-\bar{\Omega} t) / \bar{\epsilon}$, so the relevant modes are

$$
\phi \sim F(r, \theta) e^{i m \varphi-i \omega t} \sim F(r, \theta) e^{i n_{L}((\varphi-\bar{\Omega} t) / \bar{\epsilon})-i n_{R} t} \quad \text { with } \quad n_{L}=m \bar{\epsilon}, n_{R}=\omega-m \bar{\Omega}
$$

The Boltzmann factor is then

$$
e^{-\beta_{H}\left(\omega-m \Omega_{H}\right)}=e^{-\beta_{R} n_{R}-\beta_{L} n_{L}} \quad \text { with } \quad \beta_{R}=\beta_{H}, \quad \beta_{L}=\frac{\bar{\Omega}-\Omega_{H}}{\bar{\epsilon}} \beta_{H}=\beta_{H}
$$

and the entropy is thus

$$
S=\frac{\pi^{2}}{3} c T_{H}=\frac{\mathcal{A}}{4 G}
$$

as expected. The microcanonical version of the derivation is discussed in [26]; it appears to also yield the correct Bekenstein-Hawking entropy, although the zero of the conformal weight $\Delta$ is not entirely clear.

While this particular result is fairly new, it is closely related to the covariant phase space analysis of [14]. That approach has been applied to a wide variety of black holes, including dilaton black holes [68] and black holes in higher derivative theories [69,70]. As in the extremal Kerr case, the central charge depends on parameters of the black hole, and once again, black holes with different horizon areas must be described by different conformal field theories.

\subsection{Two-Dimensional Dilaton Gravity}

I will conclude this section with an interesting example of a model for which these conformal methods fail, but in an interesting way. In two spacetime dimensions, the Einstein-Hilbert Lagrangian is a total derivative, and to obtain an interesting theory of gravity, one must add new fields. The simplest choice is the Jackiw-Teitelboim model [71,72], with an action

$$
I=\frac{1}{2} \int d^{2} x \sqrt{-g} \phi\left(R+\frac{2}{\ell^{2}}\right)
$$

whose fields include both the metric and a dilaton $\phi$. The field equations obtained from this action have an asymptotically anti-de Sitter black hole solution,

$$
d s^{2}=-\left(\frac{x^{2}}{\ell^{2}}-a^{2}\right) d t^{2}+\left(\frac{x^{2}}{\ell^{2}}-a^{2}\right) d x^{2}, \quad \phi=\frac{\phi_{0} x}{\ell}
$$

with a mass

$$
M=\frac{\phi_{0} a^{2}}{2 \ell}
$$


and an entropy [73]

$$
S=\frac{2 \pi \phi_{0} a}{\ell}
$$

The asymptotic symmetries of this model were first analyzed by Hotta [74] and Cadoni and Mignemi [75,76]. The ADM constraints are

$$
H\left[\xi^{\perp}, \xi^{x}\right]=\int_{\Sigma} d x\left\{\xi^{\perp}\left[-\pi_{\phi} \pi_{\sigma}+\left(\frac{\phi^{\prime}}{\sigma}\right)^{\prime}-\lambda^{2} \sigma \phi\right]+\xi^{x}\left[\pi_{\phi} \phi^{\prime}-\pi_{\sigma}^{\prime} \sigma\right]\right\}
$$

where $\sigma=\sqrt{q_{x x}}$. In the language of Section 3 of this paper, one finds that

$$
\{\bar{H}[\xi], \bar{H}[\eta]\}=\bar{H}\left[\{\xi, \eta\}_{S D}\right]+K(\xi, \eta)
$$

with

$$
\{\xi, \eta\}_{S D}^{\perp}=\xi^{x} \eta^{\perp \prime}-\eta^{x} \xi^{\perp \prime}, \quad\{\xi, \eta\}_{S D}^{x}=\xi^{x} \eta^{x \prime}-\eta^{x} \xi^{x \prime}+\frac{1}{\sigma^{2}}\left(\xi^{\perp} \eta^{\perp \prime}-\eta^{\perp} \xi^{\perp \prime}\right)
$$

and

$$
\begin{aligned}
K(\xi, \eta)= & -B\left[\{\xi, \eta\}_{S D}\right] \\
& +\left[\left(\xi^{x} \eta^{\perp}-\eta^{x} \xi^{\perp}\right)\left(\pi_{\phi} \pi_{\sigma}+\frac{\sigma \phi}{\ell^{2}}\right)-\left(\xi^{x} \eta^{\perp \prime}-\eta^{x} \xi^{\perp \prime}\right) \frac{\phi^{\prime}}{\sigma}+\xi^{\perp} \eta^{\perp \prime}-\eta^{\perp} \xi^{\perp \prime} \frac{\pi_{\sigma}}{\sigma}\right]_{\partial \Sigma}
\end{aligned}
$$

Note that the Cauchy surface $\Sigma$ is one-dimensional, so its boundary is a discrete set of points; there is thus no integral in (84).

The asymptotic conditions

$$
g_{a b} \sim\left(\begin{array}{cc}
-\frac{x^{2}}{\ell^{2}}+\mathcal{O}(1) & \mathcal{O}\left(\frac{1}{x^{3}}\right) \\
& \frac{\ell^{2}}{x^{2}}+\mathcal{O}\left(\frac{1}{x^{4}}\right)
\end{array}\right), \quad \phi \sim \mathcal{O}(x)
$$

are preserved by the diffeomorphisms

$$
\xi^{t}=T+\frac{\ell^{4}}{2} \ddot{T}, \quad \xi^{x}=-x \dot{T}
$$

for arbitrary functions $T(t)$. The central term (84) then has a piece of the form

$$
K\left(\xi_{1}, \xi_{2}\right)=\cdots-\ell \phi_{0}\left(\dot{T}_{1} \ddot{T}_{2}-\dot{T}_{2} \ddot{T}_{1}\right)
$$

This is almost the right form for the central extension of a Virasoro algebra. Indeed, as Cadoni and Mignemi pointed out, if one were to arbitrarily introduce an integral over $t$ with period $2 \pi \ell$, the period of Euclidean AdS space, one would find a central charge

$$
c=24 \phi_{0}
$$

This is too large by a factor of two: for instance, the temperature of the black hole (78) is

$$
T_{H}=\frac{a}{2 \pi \ell}
$$


and the canonical Cardy formula would give

$$
S=\frac{\pi^{2}}{3} c T=\frac{4 \pi \phi_{0} a}{\ell}
$$

twice the entropy (80). One can also compare (88) with the central charge (72) of the preceding section, noting that the dimensional reduction of $(3+1)$-dimensional spherically symmetric gravity gives dilaton gravity with a dilaton $\phi=\mathcal{A} / 8 \pi G$. Again, an extra factor of two is present.

It is plausible that this factor of two problem has a good solution. For example, although the vector fields (86) obey a standard Diff $S^{1}$ algebra, the corresponding surface deformation algebra (83) contains three-derivative terms, so the separation of "central" and "boundary" terms in (84) depends on details of the choice of $B[\xi]$ (see, for instance, [77]). The integral over time, on the other hand, is more mysterious, although a similar time averaging has recently been suggested for the $\mathrm{AdS}_{2} / \mathrm{CFT}_{1}$ correspondence in a rather different context [78].

There are other ways to obtain a dual conformal field theory in the $(1+1)$-dimensional case. For example, one can use the AdS/CFT correspondence to identify the boundary stress-energy tensor and look at its anomalous transformation properties [79-81]. One can also lift the theory to $2+1$ dimensions and exploit the known properties of the BTZ black hole [80-82]; for the extremal case, this may offer an interesting connection to the extremal Kerr/CFT correspondence described in Section 4.2, and might explain the appearance of a chiral CFT [83]. These approaches are designed rather particularly for the $(1+1)$-dimensional black hole, however, and seem less general than the boundary symmetry method I have focused on in this review.

\section{What Does This Tell Us about the States?}

One of the chief attractions of the dual conformal field theory approach to black hole thermodynamics is its universality. The computations I have described depend on only a few minimal features of the black hole; understanding the asymptotic or near-horizon symmetry is already enough to determine the entropy. But this virtue is also a weakness: it means that the thermodynamics may tell us relatively little about the true microscopic degrees of freedom of the black hole.

An analogous situation occurs in high energy theory [84]. When a global symmetry is spontaneously broken, Goldstone's theorem tells us that there must be a corresponding set of massless excitations [85]. One such Goldstone boson occurs for each "broken" symmetry generator, and can be viewed as an excitation along the "would-be symmetry" that is broken by the vacuum. But while Goldstone's theorem gives us some information about these massless bosons, it does not tell us how they are constructed in terms of the elementary quanta of the theory.

This analogy can be strengthened. The fundamental symmetry of general relativity is diffeomorphism invariance. This is a gauge symmetry, not a global symmetry of the type directly relevant to Goldstone bosons. Still, it remains true that when the symmetry is broken by the imposition of boundary conditions, "would-be pure gauge" degrees of freedom-excitations that would normally be pure gauge, but that do not respect the boundary conditions_-become physical at the boundary [86,87].

More quantitatively, in Dirac quantization, physical states should be annihilated by the constraints $H[\xi]$. But if the central term $K[\xi, \eta]$ in (8) is nonzero, one cannot consistently require such a condition. For conformal field theories in two dimensions, this issue is well known [41]; one may instead require, 
for example, that only the positive frequency parts of the Virasoro generators annihilate physical states. The result is that certain states that would otherwise be considered unphysical must now be allowed. Since the central term is nonzero only at the boundary, these new states are naturally associated with the boundary, offering an explanation for the fact that black hole entropy depends on area rather than volume.

I cannot say for certain that this is a full explanation for the success of the dual CFT approach. There is one case, though, in which the issue has been investigated more carefully [88]. In $(2+1)$-dimensional asymptotically anti-de Sitter space, the complete set of boundary terms for the Einstein-Hilbert action is known explicitly. These include terms that are not invariant under diffeomorphisms normal to the boundary. If one decomposes the metric near the boundary into a gauge-fixed piece and a "pure gauge" piece, one can determine the induced action for the "pure gauge" portion. The result is a conformal field theory (a Liouville theory) with a central charge that exactly matches the expression (27) for the BTZ black hole. The relevant states live in the "nonnormalizable sector" of the theory, whose quantization is unclear (though see [89]), but this result seems to support the picture of black hole states as "would-be pure gauge" degrees of freedom quite nicely.

\section{Conclusions}

In general, black holes are neither two-dimensional nor conformally invariant. Nevertheless, we have seen that black hole thermodynamics often has a simple description in terms of a two-dimensional conformal field theory at a suitable boundary. I have focused on the computation of the most important thermodynamic quantity, the Bekenstein-Hawking entropy, but similar methods can be employed to analyze greybody factors and superradiance [51,52]. Similarly, a two-dimensional conformal field theory approach to matter near a black hole horizon can be used to extract the Hawking temperature and spectrum [90-96].

While these are important steps, more remains to be done. A complete dual description of black hole thermodynamics should also include Hawking radiation, which would presumably require a coupling of the boundary CFT to bulk matter. For the BTZ black hole, an important step in this direction was taken by Emparan and Sachs [53], who considered an external field coupled to the dual CFT and showed that one could derive the Hawking spectrum from detailed balance. Their method can probably be extended to other types of black holes, although as far as I know this has not yet been done.

For some important problems in black hole thermodynamics-the information loss puzzle, for instance [97], and the question of the final state of Hawking evaporation-one must go even further, and account for the back-reaction of Hawking radiation on the black hole. As noted above, this probably cannot be accomplished within the framework of a single conformal field theory: the central charge of the dual CFT typically depends on quantities such as the horizon area or angular momentum that change evaporation. One can imagine a picture involving a flow from an initial CFT to a final one-indeed, in the examples of Section 4, the direction of such a flow is compatible with Zamolodchikov's $c$ theorem [98]—but for now, this possibility remains speculative. 


\section{Acknowledgements}

Portions of this project were carried out at the Peyresq 15 Physics Conference with the support of OLAM Association pour la Recherche Fundamentale, Bruxelles. This work was supported in part by Department of Energy grant DE-FG02-91ER40674.

\section{References and Notes}

1. Bekenstein, J.D. Black holes and entropy. Phys. Rev. 1973, D7, 2333-2346.

2. Hawking, S.W. Black hole explosions. Nature 1974, 248, 30-31.

3. Iyer, V.; Wald, R.M. Some properties of Noether charge and a proposal for dynamical black hole entropy. Phys. Rev. 1994, D50, 846-864.

4. Carlip, S. Black hole thermodynamics and statistical mechanics. In Physics of Black Holes: A Guided Tour; Papantonopoulos, E., Ed.; Springer: Berlin, Germany, 2009; pp. 89-123.

5. Carlip,S. Statistical mechanics and black hole entropy. In Field Theory, Integrable Systems and Symmetries; Khanna, F., Vinet, L., Eds.; Les Publications CRM: Montreal, Canada, 1997; pp. 11-19.

6. Banados, M.; Teitelboim, C; Zanelli, J. The black hole in three-dimensional space-time. Phys. Rev. Lett. 1992, 69, 1849-1851.

7. Strominger, A. Black hole entropy from near horizon microstates. J. High Energy Phys. 1998, 9802, 009:1-009:11.

8. Birmingham, D.; Sachs, I.; Sen, S. Entropy of three-dimensional black holes in string theory. Phys. Lett. 1998, B424, 275-280.

9. Carlip, S. Conformal field theory, (2+1)-dimensional gravity, and the BTZ black hole. Classical Quantum Gravity 2005, 22, R85-R124.

10. Witten, E. Three-dimensional gravity revisited, 2007. arXiv:0706.3359. arXiv.org e-Print archive. Available online: http://arXiv.org/abs/arXiv:0706.3359 (accessed on 15 July 2011).

11. Maloney, A.; Witten, E. Quantum gravity partition functions in three dimensions. J. High Energy Phys. 2010, 1002, 029:1-029:58.

12. Carlip, S. Black hole entropy from conformal field theory in any dimension. Phys. Rev. Lett. 1999, 82, 2828-2831.

13. Solodukhin, S.N. Conformal description of horizon's states. Phys. Lett. 1999, B454, 213-222.

14. Carlip, S. Entropy from conformal field theory at Killing horizons. Classical Quantum Gravity 1999, 16, 3327-3348.

15. Guica, M.M.; Hartman, T.; Song, W.; Strominger, A. The Kerr/CFT correspondence. Phys. Rev. 2009, D80 124008:1-124008:9.

16. Cardy, J.L. Operator content of two-dimensional conformally invariant theories. Nucl. Phys. 1986, B 270, 186-204.

17. Blöte, H.W.J.; Cardy, J.L.; Nightingale, M.P. Conformal invariance, the central charge, and universal finite size amplitudes at criticality. Phys. Rev. Lett. 1986, 56, 742-745.

18. Carlip, S. Horizon constraints and black hole entropy. Classical Quantum Gravity 2005, 22, 1303-1312. 
19. Carlip, S. Black hole thermodynamics from Euclidean horizon constraints. Phys. Rev. Lett. 2007, 99, 021301:1-021301:4.

20. Brown, J. D.; Henneaux, M. Central charges in the canonical realization of asymptotic symmetries: An example from three-dimensional gravity. Commun. Math. Phys. 1986, 104, 207-226.

21. Skenderis, K. Black holes and branes in string theory. Lect. Notes Phys. 2000, 541, 325-364.

22. Bardeen, J; Horowitz, G.T. The extreme Kerr throat: A vacuum analog of $\mathrm{AdS}_{2} \times S^{2}$. Phys. Rev. 1999, D60, 104030:1-10403:10.

23. Carlip, S.; Clements, M.; Della Pietra,S.; Della Pietra, V. Sewing Polyakov amplitudes I: Sewing at a fixed conformal structure. Commun. Math. Phys. 1990, 127, 253-271.

24. Witten, E. On holomorphic factorization of WZW and coset models. Commun. Math. Phys. 1992, 144, 189-212.

25. Thorne,K.S.; MacDonald, D.A.; Price, R.H. Black Holes: The Membrane Paradigm; Yale University Press: New Haven, CT, USA, 1986.

26. Carlip, S. Extremal and nonextremal Kerr/CFT correspondences. J. High Energy Phys. 2011, 1104, 076:1-076:17.

27. Kiefer, C. Quantum Gravity; Clarendon Press: Oxford, UK, 2004.

28. Wald, R.M. Black hole entropy is the Noether charge. Phys. Rev. 1993, D48, 3427-3431.

29. Ashtekar, A.; Bombelli, L.; Reula, O. The covariant phase space of asymptotically flat gravitational fields. In Mechanics, Analysis and Geometry: 200 Years after Lagrange; Francaviglia, M., Ed.; North-Holland: Amsterdam, The Netherlands, 1991; pp. 417-450.

30. Crnkovic, C.; Witten, E. Covariant description of canonical formalism in geometrical theories. In Three Hundred Years of Gravitation; Hawking, S.W., Israel, W., Eds.; Cambridge University Press: Cambridge, UK, 1987; pp. 676-684.

31. Arnowitt, R.; Deser, S.; Misner, C.W. The dynamics of general relativity. In Gravitation: an introduction to current research; Witten, L., Ed.; Wiley: New York, NY, USA, 1962; pp. 227-265.

32. Teitelboim, C. How commutators of constraints reflect the space-time structure. Ann. Phys. 1973, $79,542-557$.

33. Guven, J.; Ryan, M.P. Functional integrals and canonical quantum gravity: Facts and fancies. Phys. Rev. 1992, D45, 3559-3576.

34. Regge, T.; Teitelboim, C. Role of surface integrals in the Hamiltonian formulation of general relativity. Ann. Phys. 1974, 88, 286-318.

35. Solovev, V.O. Boundary values as Hamiltonian variables. I. New Poisson brackets. J. Math. Phys. 1993, 34, 5747-5769.

36. Koga, J. Asymptotic symmetries on killing horizons. Phys. Rev. 2001, D64, 124012:1-124012:19.

37. Silva, S. Black hole entropy and thermodynamics from symmetries. Classical Quantum Gravity 2002, 19, 3947-3962.

38. Barnich, G.; Brandt, F. Covariant theory of asymptotic symmetries, conservation laws and central charges. Nucl. Phys. 2002, B 633, 3-82.

39. Compere, G. Symmetries and conservation laws in Lagrangian gauge theories with applications to the mechanics of black holes and to gravity in three dimensions. arXiv:0708.3153. arXiv.org e-Print archive. Available online: http://arXiv.org/abs/arXiv:0708.3153 (accessed on 15 July 2011). 
40. Gel'fand, I.M.; Fuks, D.B. Cohomology of Lie algebra of the vector fields on the circle. Funct. Anal. Appl. 1968, 2, 342-343.

41. Di Francesco, P.; Mathieu, P.; Sénéchal, D. Conformal Field Theory; Springer: New York, NY, USA, 1997.

42. Rasmussen, J. A near-NHEK/CFT correspondence. arXiv:1004.4773. arXiv.org e-Print archive. Available online: http://arXiv.org/abs/arXiv:1004.4773 (accessed on 15 July 2011).

43. Ashtekar, A.; Krishnan, B. Isolated and dynamical horizons and their applications. Living Rev. Relativ. 2004, 7, 10:1-10:91.

44. Date, G. Isolated horizon, Killing horizon, and event horizon. Classical Quantum Gravity 2001, $18,5219-5226$.

45. Wall, A.C. A proof of the generalized second law for rapidly changing fields and arbitrary horizon slices. arXiv:1105.3445. arXiv.org e-Print archive. Available online: http://arXiv.org/abs/arXiv:1105.3445 (accessed on 15 July 2011).

46. Carlip, S. Logarithmic corrections to black hole entropy from the Cardy formula. Classical Quantum Gravity 2000, 17, 4175-4186.

47. Birmingham, D.; Sachs, I.; and Sen, S. Exact results for the BTZ black hole. Int. J. Mod. Phys. 2001, D10, 833-858.

48. Loran, F.; Sheikh-Jabbari, M.M.; Vincon, M. Beyong logarithmic corrections to Cardy formula. J. High Energy Phys. 2011, 1101, 110:1-110:26.

49. Carlip, S. What we don't know about BTZ black hole entropy. Classical Quantum Gravity 1998, 15, 3609-3625.

50. Bousso, R.; Maloney, A.; and Strominger, A. Conformal vacua and entropy in de Sitter space. Phys. Rev. 2002, D65, 104039:1-104039:24.

51. Maldacena, J.M.; Strominger, A. Universal low-energy dynamics for rotating black holes. Phys. Rev. 1997, D56, 4975-4983.

52. Bredberg, I.; Hartman, T.; Song, W.; Strominger, A. Black hole superradiance from Kerr/CFT. J. High Energy Phys. 2010, 019:1-019:32.

53. Emparan, R.; Sachs, I. Quantization of $\mathrm{AdS}_{3}$ black holes in external fields. Phys. Rev. Lett. 1998 $81,2408-2411$.

54. I use the sign conventions of [99], and units $16 \pi G=1$, although I will occasionally restore factors of $G$.

55. Brown, J.D.; Lau, S.R.; York, J.W. Action and energy of the gravitational field. Ann. Phys. 2002 297, 175-218.

56. Bañados, M.; Henneaux, M.; Teitelboim, C.; Zanelli, J. Geometry of the $2+1$ black hole. Phys. Rev. 1993, D4, 1506-1525.

57. The full AdS/CFT correspondence involves many additional degrees of freedom; it remains unclear whether $(2+1)$-dimensional gravity alone contains enough degrees of freedom to fully account for black hole entropy [9-11].

58. Bredberg, I.; Keeler, C.; Lysov, V.; Strominger, A. Cargese lectures on the Kerr/CFT correspondence. arXiv:1103.2355. arXiv.org e-Print archive. Available online: http://arXiv.org/ abs/arXiv:1103.2355 (accessed on 15 July 2011). 
59. Frolov, V.P.; Novikov, I.D. Black Hole Physics; Kluwer: Dordrecht, The Netherlands, 1998.

60. Castro, A.; Maloney, A.; Strominger, A. Hidden conformal symmetry of the Kerr black hole. Phys. Rev. 2010 D82, 024008:1-024008:7.

61. Frolov, V.P.; Thorne, K.S. Renormalized stress-energy tensor near the horizon of a slowly evolving, rotating black hole. Phys. Rev. 1989 , D39, 2125-2154.

62. Hartman, T.; Murata, K.; Nishioka, T.; Strominger, A. CFT duals for extreme black holes. J. High Energy Phys. 2009, 0904, 019:1-019:17.

63. Lu, H.; Mei, J.; Pope, C.N. Kerr/CFT correspondence in diverse dimensions. J. High Energy Phys. 2009, 0904, 054:1-054:17.

64. Castro, A.; Larsen, F. Near extremal Kerr entropy from $\mathrm{AdS}_{2}$ quantum gravity. J. High Energy Phys. 2009, 0912, 037:1-037:24.

65. Hawking, S.W. Black holes in general relativity. Commun. Math. Phys. 1972, 25, 152-166.

66. Medved, A.J.M.; Martin, D.; Visser, M. Dirty black holes: Symmetries at stationary nonstatic horizons. Phys. Rev. 2004, D70, 024009:1-024009:8.

67. Here and in what follows, there is a subtlety regarding the radial derivatives $\partial_{\rho}$, coming from the fact that the proper distance $\rho$ is metric-dependent. This issue is discussed in detail in the appendices of [26].

68. Jing J.; Yan, M.L. Statistical entropy of the static dilaton black holes from the Cardy formulas. Phys. Rev. 2001, D63, 024003:1-024003:6.

69. Cvitan, M.; Pallua, S.; Prester, P. Entropy of Killing horizons from Virasoro algebra in D-dimensional extended Gauss-Bonnet gravity. Phys. Lett. 2003, B555, 248-254.

70. Cvitan, M.; Pallua, S. Conformal entropy for generalised gravity theories as a consequence of horizon properties. Phys. Rev. 2005, D71, 104032:1-104032:9.

71. Jackiw, R. Liouville theory: a two-dimensional model for gravity? In Quantum Theory of Gravity; Christensen, S.M., Ed.; Adam Hilger Ltd.: Bristol, UK, 1984; pp. 403-420.

72. Teitelboim, C. The Hamiltonian structure of two-dimensional space-time and its relation with the conformal anomaly. In Quantum Theory of Gravity; Christensen, S.M., Ed.; Adam Hilger Ltd.: Bristol, UK, 1984; pp. 327-344.

73. Cadoni, M.; Mignemi, S. Nonsingular four-dimensional black holes and the Jackiw-Teitelboim theory. Phys. Rev. 1995, D51, 4319-4329.

74. Hotta, M. Asymptotic isometry and two-dimensional anti-de Sitter gravity. arXiv:gr-qc/9809035. arXiv.org e-Print archive. Avalable online: http://arXiv.org/abs/arXiv:gr-qc/9809035 (accessed on 15 July 2011).

75. Cadoni, M.; Mignemi, S. Entropy of 2-D black holes from counting microstates. Phys. Rev. 1999, D59, 081501:1-081501:5.

76. Cadoni, M.; Mignemi, S. Asymptotic symmetries of $\mathrm{AdS}_{2}$ and conformal group in $d=1$. Nucl. Phys. 1999, B557, 165-180.

77. Catelani, G.; Vanzo, L. On the $2^{1 / 2}$ puzzle in $\mathrm{AdS}_{2} / C F T_{1}$. arXiv:hep-th/0009186. arXiv.org e-Print archive. Avalable online: http://arXiv.org/abs/arXiv:hep-th/0009186 (accessed on 15 July 2011). 
78. Chamon, C.; Jackiw, R.; Pi, S.-Y.; Santos, L. Conformal quantum mechanics as the CFT 1 dual to $\mathrm{AdS}_{2}$. Phys. Lett. 2011, B70, 503-507.

79. Navarro-Salas, J.; Navarro, P. $\mathrm{AdS}_{2} / \mathrm{CFT}_{1}$ correspondence and near extremal black hole entropy. Nucl. Phys. 2000, B579, 250-266.

80. Castro, A.; Grumiller, D.; Larsen, F.; McNees, R. Holographic description of $\mathrm{AdS}_{2}$ black holes. J. High Energy Phys. 2008, 0811, 052:1-052:28.

81. Castro, A.; Keeler, C.; Larsen, F. Three dimensional origin of $\mathrm{AdS}_{2}$ gravity. J. High Energy Phys. 2010, 1007, 033:1-033:25.

82. Izquierdo, J.M.; Navarro-Salas, J.; Navarro, P. Kaluza-Klein theory, AdS/CFT correspondence and black hole entropy. Classical Quantum Gravity 2002, 19, 563-570.

83. Balasubramanian,V.; de Boer, J.; Sheikh-Jabbari, M.M.; Simon, J. What is a chiral 2d CFT? And what does it have to do with extremal black holes? J. High Energy Phys. 2010, 1002, 017:1-017:20.

84. This analogy was suggested to me by Nemanja Kaloper and John Terning.

85. Goldstone, J.; Salam, A.; Weinberg, S. Broken symmetries. Phys. Rev. 1962, 127, 965-970.

86. Carlip, S. Statistical mechanics and black hole thermodynamics. In Constrained Dynamics and Quantum Gravity 1996; de Alfaro, V., Nelson, J.E., Bandelloni, G., Blasi, A., Cavaglia, M., Filippov, A.T., Eds.; North-Holland: Amsterdam, The Netherlands, 1997; pp. 8-12.

87. Carlip, S. Symmetries, horizons, and black hole entropy. Gen. Rel. Grav. 2007, 39, 1519-1523.

88. Carlip, S. Dynamics of asymptotic diffeomorphisms in (2+1)-dimensional gravity. Classical Quantum Gravity 2005, 22, 3055-3060.

89. Chen, Y. Quantum Liouville theory and BTZ black hole entropy. Classical Quantum Gravity 2004, 21, 1153-1180.

90. Robinson, S.P.; Wilczek, F. A relationship between Hawking radiation and gravitational anomalies. Phys. Rev. Lett. 2005, 95, 011303:1-011303:4.

91. Iso, S.; Umetsu, H.; Wilczek, F. Hawking radiation from charged black holes via gauge and gravitational anomalies. Phys. Rev. Lett. 2006, 96, 151302:1-151302:4.

92. Banerjee, R.; Kulkarni, S. Hawking radiation and covariant anomalies. Phys. Rev. 2008, D77, 024018:1-024018:5.

93. Iso, S.; Morita, T.; Umetsu, H. Hawking radiation via higher-spin gauge anomalies. Phys. Rev. 2008, D 77, 045007:1-045007:8.

94. Bonora, L.; Cvitan, M.; Pallua, S.; Smolić, I. Hawking radiation, $W_{\infty}$ algebra and trace anomalies. J. High Energy Phys. 2008, 0805, 071:1-071:22.

95. Bonora, L.; Cvitan, M.; Pallua, S.; Smolić, I. Hawking fluxes, $W_{\infty}$ algebras and anomalies. J. High Energy Phys. 2008, 0812, 021:1-021:32.

96. Bonora, L.; Cvitan, M.; Pallua, S.; Smolić, I. Hawking fluxes, fermionic currets, $W_{1+\infty}$ algebras and anomalies. Phys. Rev. 2009, D80, 084034:1-084034:12.

97. Giddings, S.B. The black hole information paradox. arXiv:hep-th/9508151. arXiv.org e-Print archive. Available online: http://arXiv.org/abs/arXiv:hep-th/9508151 (accessed on 15 July 2011).

98. Zamolodchikov, A.B. Irreversibility of the flux of the renormalization group in a 2D field theory. JETP Lett. 1986, 43, 730-732. 
99. Wald, R.M. General Relativity; University of Chicago Press: Chicago, IL, USA, 1984.

(c) 2011 by the authors; licensee MDPI, Basel, Switzerland. This article is an open access article distributed under the terms and conditions of the Creative Commons Attribution license (http://creativecommons.org/licenses/by/3.0/.) 